\title{
Comparison of Genetic Diversity Based on SSR Markers Between Peanut Mini Core Collections from China and ICRISAT
}

\author{
JIANG Hui-Fang ${ }^{1, *}$, REN Xiao-Ping ${ }^{1}$, ZHANG Xiao-Jie ${ }^{1}$, HUANG Jia-Quan ${ }^{1}$, LEI Yong ${ }^{1}$, YAN \\ Li-Ying ${ }^{1}$, LIAO Bo-Shou ${ }^{1}$, Hari D. UPADHYAYA ${ }^{2}$, and Corley C. HOLBROOK ${ }^{3}$ \\ ${ }^{1}$ Oil Crops Research Institute of Chinese Academy of Agricultural Sciences/ Key Laboratory of Oil Crop Biology of Ministry of Agriculture, \\ Wuhan 430062, China \\ ${ }^{2}$ International Crops Research Institute for the Semiarid Tropics, Patancheru, AP 502 324, India \\ ${ }^{3}$ USDA-ARS, P.O. Box 748, Tifton, Georgia 31793, USA
}

\begin{abstract}
Mini core collection plays an important role in evaluating genetic resources of peanut (Arachis hypogaea L.). This study aimed at comparing the genetic diversities of a domestic and exotic peanut mini core collections and providing basic data for germplasm introduction and peanut breeding. The exotic mini core collection, which was introduced from the International Crops Research Institute for the Semi-Arid Tropics (ICRISAT, Patancheru, India), consisted of 168 accessions, whereas the Chinese mini core collection consisted of 298 accessions. A total of 26 polymorphic simple sequence repeat (SSR) markers were used to evaluate the genetic diversity of these genotypes and their similarities. The similarity coefficient ranged from 0.49 to 0.99 among the total 466 accessions and the largest genetic distance was between "L2 Gangguo" from the Chinese core collection and "ICG12625" from the ICRISAT core collection. Among the 6 botanical types of peanut, the fastigiata type had the largest genetic diversity indices in both Chinese and ICRISAT mini core collections, which were 1.11 and 0.97 , respectively. The hypogaea type had the second largest diversity with genetic diversity indices of 0.89 and 0.88 for Chinese and ICRISAT mini core collections, respectively. The genetic distance between the 2 mini core collections was larger than that within a mini core collection. Particularly, the aequatoriana genotype ICG12625 from ICRISAT had the largest differences to the Chinese genotypes. According to similarity coefficient and genetic diversity index, the genetic diversity was greater among genotypes from the Chinese mini core collection than that from the ICRISAT mini core collection.
\end{abstract}

Keywords: peanut; core collection; SSR marker; genetic diversity

In the last three decades, genetic improvement has made significant progresses in peanut (Arachis hypogaea L.) production in China ${ }^{[1-4]}$. Among over 200 peanut cultivars developed, at least 70\% were associated with Fuhuasheng and Shitouqi, which were landraces with elite agronomic traits ${ }^{[5-7]}$. Therefore, the commercial peanut cultivars have a very narrow genetic basis, and plenty of genetic variability implicated in peanut germplasm has been ignored in breeding programs. At present, 7337 accessions of peanut germplasm are preserved in the GenBank, including native and introduced varieties and wild relatives ${ }^{[8-10]}$. The poor utilization of these resources is mainly because of the scant knowledge on their genetic diversity and genetic basis for elite gene or trait ${ }^{[11-15]}$. Evaluation of genetic diversity is a basic work to explain the genetic relations among variety types or germplasm sources and to reveal the inheritability mechanism of potential elite genes. Besides, distinct germplasm with favorable genes may be discovered in these basic studies, which plays important roles in background wideness and cultivar improvement ${ }^{[16-19]}$.

Core collection, which is defined as a subset of accessions from the entire collection that capture most of available

Received: 25 December 2009; Accepted: 20 April 2010.

* Corresponding author. E-mail: xuzhengjin@126.com.

Copyright (C) 2010, Crop Science Society of China and Institute of Crop Sciences, Chinese Academy of Agricultural Sciences. Published by Elsevier BV. All rights reserved. Chinese edition available online at http://www.chinacrops.org/zwxb/

DOI: $10.1016 / \mathrm{S} 1875-2780(09) 60059-6$ 
genetic diversity of the species, is regarded as an optimal population in evaluating genetic diversity and mining elite genes ${ }^{[20-22]}$. The International Crops Research Institute for the Semi-Arid Tropics (ICRISAT, Patancheru, India) has established a mini core collection of peanut, which consists of 184 accessions ${ }^{[12,13]}$. In China, the peanut mini core collection is composed of 298 accessions ${ }^{[9,10]}$. The ICRISAT genebank preserves a total of 14,889 peanut accessions from 93 countries, but only a few from China ${ }^{[20]}$. In Chinese collections, the exotic germplasms are introduced from 32 countries and ICRISAT. Due to the reintroduction of peanut germplasm across countries and ICRISAT (sometimes the same material possesses different names in different countries), the duplicates of germplasm may interfere the accurate evaluation of genetic diversity in a population. In mini core collection subsets with various botanical types of peanut, almost all phenotypic variations in the basic collection are presented with enough diversity. Therefore, we took the mini core collections from China and ICRISAT as experimental materials to evaluate and compare their genetic diversities and similarity using simple sequence repeat (SSR) markers. The objectives of this study were to provide guidance to the effective utilization of Chinese peanut resources and the principles for germplasm introduction.

\section{Materials and methods}

\subsection{Plant materials and DNA extraction}

The Chinese mini core collection contained 298 accessions, including 29 accessions of fastigiata type, 119 accessions of vulgaris type, 21 accessions of hirsuta type, 110 accessions of hypogaea type, and 19 accessions of intermediate type. The ICRISAT mini core collection was composed of 168 accessions in fastigiata (30 accessions), vulgaris (64 accessions), hypogaea (71 accessions), peruviana (2 accessions), and aquatoriana (1 accession) types.

Genomic DNA of each genotype was extracted from young leaves using cetyltrimethylammonium bromide (CTAB) method ${ }^{[14,15]}$. The quality of DNA extracted was validated through fluorescence test (DU-650 fluorometer, Beckman, California, USA) and electrophoresis in $1 \%$ agarose gels.

\subsection{SSR analysis}

A total of 204 pairs of SSR primers were used to screen polymorphic primers. The sequences of these primers were provided by the Biotechnology Laboratory of ICRISAT and synthesized by the Shanghai Sangon Biological Engineering Technology Services Co. Ltd. (Shanghai, China). PCR reaction mixture was $10 \mu \mathrm{L}$ in volume consisting of $1 \times$ buffer, $0.4 \mathrm{mmol} \mathrm{L}{ }^{-1} \mathrm{dNTPs}, 0.3 \mathrm{pmol} \mathrm{L}{ }^{-1}$ primers, $2.0 \mathrm{mmol} \mathrm{L}^{-1}$ $\mathrm{Mg}^{2+}, 0.5 \mathrm{U}$ Taq DNA polymerase (Fermentas, Vilnius Lithuania), and 15 ng DNA. PCR amplification was carried out in an MJ Research Thermocycler (PTC-100, Waltham, USA) as described in our earlier reports ${ }^{[14,15]}$. The amplified products were separated in $6 \%$ denaturing polyacrylamide gels (PAGE) and visualized after silver staining ${ }^{[16]}$.

\subsection{Data analysis}

The amplified fragments at a locus were scored as " 1 " for allele presence and " 0 " for allele absence. Shannon-Weaver index $(I)$ was calculated using Popgen Ver.1.32 ${ }^{[17,18]}$. The genetic relationships among genotypes were analyzed using NTSYS-PCV.2.0 based on similarity coefficient and 3-dimension principal component analysis (3D-PCA) ${ }^{[17,18]}$. Polymorphic information content (PIC) was calculated using the following formula ${ }^{[19]}$ :

$$
P I C=1-\sum_{i=1}^{k} P_{i}^{2}
$$

Where, $i$ is the number of loci, $k$ is the number of alleles at a locus, and $P_{i}$ is the allelic frequency at the $i$ th locus.

\section{Results}

\subsection{Polymorphic bands in Chinese and ICRISAT mini core collections}

Twenty-four accessions in different botanical types were selected to screen polymorphic primers, and 54 pairs of SSRs presented polymorphism. These SSR primers were amplified in the total of 466 accessions and 26 pairs of them generated clear bands. The PCR products were designated with marker name and fragment size. For example, "2A06/440" stands for the $440 \mathrm{bp}$ fragment at 2A06 locus. This fragment was detected only in 21 genotypes of hypogaea type and 2 genotypes of hirsuta type from Chinese core collection, but absent in genotypes from ICRISAT mini core collection and vulgaris and fastigiata types from Chinese core collection. Another 4 fragments were also observed only in a few Chinese accessions: $2 \mathrm{~B} 10 / 300$ in a unique accession of vulgaris type, 3B08/440 and 18B08/340 in 2 accessions of hypogaea type, and 9E08/560 in 2 accessions of hypogaea type and 32 accessions of vulgaris type. This result indicates that Chinese collection had more banding patterns than the ICRISAT collection.

Some fragments were presented in a few specific botanical types. For instance, PM443/270 was only found in 5 accessions of fastigiata type and 5 accessions of vulgaris type, but absent in the ssp. hypogaea (hypogaea and hirsuta types); $3 \mathrm{D} 09 / 380,3 \mathrm{D} 09 / 450$, and $2 \mathrm{D} 12 \mathrm{~B} / 440$ in 3,3 , and 1 accessions of hypogaea type, respectively; 9E08/550 and $16 \mathrm{C} 06 / 430$ in 6 and 1 accessions of fastigiata type, respectively. This implies that some SSR allele bands are specific to certain botanical types of peanut.

The polymorphic primers detected $2-8$ bands at each locus, 
of which 17E01, 16C06, PM443, 14H06, 8D09, and 7G02 were primers in high efficiency ( $6-8$ bands each). The average numbers of bands were 2.3-4.0 for the 5 types in the Chinese collection and 1.1-3.8 for the 4 types in the ICRISAT collection. The band numbers were larger in fastigiata, vulgaris, and hypogaea types of the Chinese collection than those of the ICRISAT collection (Table 1). Although peruviana and aequatoriana types were present in the ICRISAT collection and absent in the Chinese collection, no specific banding pattern was detected in genotypes of the both types. In contrast, hirsuta type was only involved in the Chinese collection, and a specific band, 2A06/440, was amplified in 2 genotypes of hirsuta type.

\subsection{PIC values in Chinese and ICRISAT mini core collections}

Among the 26 polymorphic primers, 17E01, 16C06, PM443, 14H06, 8D09, 7G02, 1B09, 13A10, PM137, and 2E06 showed high total PIC values, which ranged from 0.896 to 0.989 . The PIC values were $0.761,0.719$, and 0.679 in fastigiata, hypogaea, and vulgaris types from the Chinese collection, respectively; and they were $0.708,0.665$, and 0.663 in the 3 types from the ICRISAT collection, respectively (Table 2). Generally, the Chinese mini core collection had a larger diversity than the ICRISAT mini core collection according to PIC. This is in agreement with the result based on polymorphic bands.

\subsection{Similarity coefficient and genetic diversity index in mini core collections from China and ICRISAT}

The similarity coefficient between a genotype from the Chinese mini core collection and a genotype from the ICRISAT mini core collection ranged from 0.49 to 0.99 . There were 2791 genotype pairs with low similarity (similarity coefficient $\leq 0.60$ ), which accounted for $2.56 \%$ of the total 466 accessions. Although the number of genotype pair with similarity in the Chinese collection (560 pairs) was larger than that in the ICRISAT collection (367 pairs), this percentage was $1.26 \%$ for the former and $2.59 \%$ for the latter. The average genetic diversity indices were 0.97 for the Chinese collection and 0.89 for the ICRISAT collection (Table 3 ). These results indicate that the genetic diversity is larger in the Chinese

Table 1 Number of polymorphic band detected in peanut types from Chinese and ICRISAT mini core collections based on 26 SSR primers

\begin{tabular}{|c|c|c|c|c|c|c|c|c|c|}
\hline \multirow{2}{*}{ Primer } & \multicolumn{5}{|c|}{ Mini core collection from China } & \multicolumn{4}{|c|}{ Mini core collection from ICRISAT* } \\
\hline & fastigiata & vulgaris & hirsuta & hypogaea & Intermediate & fastigiata & vulgaris & peruviana & hypogaea \\
\hline 2A06 & 2 & 2 & 3 & 3 & 2 & 2 & 2 & 2 & 3 \\
\hline 2B10 & 3 & 4 & 2 & 0 & 2 & 3 & 3 & 2 & 3 \\
\hline 2E06 & 4 & 4 & 4 & 4 & 4 & 4 & 4 & 0 & 4 \\
\hline PM436 & 3 & 3 & 2 & 4 & 4 & 4 & 4 & 0 & 4 \\
\hline PM137 & 5 & 5 & 5 & 5 & 2 & 5 & 5 & 4 & 5 \\
\hline $18 \mathrm{C} 05$ & 4 & 4 & 3 & 4 & 2 & 4 & 4 & 0 & 3 \\
\hline $13 \mathrm{~A} 07$ & 2 & 2 & 0 & 2 & 2 & 2 & 0 & 0 & 2 \\
\hline 9E08 & 4 & 4 & 0 & 4 & 2 & 3 & 4 & 2 & 3 \\
\hline $19 \mathrm{~A} 05$ & 2 & 2 & 0 & 2 & 0 & 2 & 2 & 2 & 2 \\
\hline $15 \mathrm{~F} 12$ & 3 & 3 & 3 & 3 & 2 & 3 & 3 & 0 & 3 \\
\hline 3D09 & 0 & 0 & 0 & 4 & 0 & 2 & 0 & 0 & 4 \\
\hline 7H06 & 2 & 3 & 0 & 2 & 2 & 2 & 2 & 0 & 2 \\
\hline $13 \mathrm{~A} 10$ & 4 & 4 & 0 & 4 & 0 & 4 & 4 & 0 & 4 \\
\hline 18B08 & 4 & 4 & 0 & 5 & 0 & 4 & 0 & 0 & 4 \\
\hline 17E01 & 6 & 6 & 4 & 6 & 0 & 6 & 6 & 2 & 4 \\
\hline $16 \mathrm{C} 06$ & 8 & 6 & 4 & 6 & 5 & 8 & 5 & 2 & 6 \\
\hline $15 \mathrm{D} 03$ & 3 & 3 & 3 & 3 & 3 & 3 & 3 & 2 & 3 \\
\hline $16 \mathrm{~F} 10$ & 3 & 3 & 3 & 3 & 3 & 2 & 2 & 1 & 2 \\
\hline PM443 & 6 & 6 & 5 & 5 & 4 & 4 & 6 & 0 & 4 \\
\hline $14 \mathrm{H} 06$ & 6 & 6 & 4 & 6 & 4 & 6 & 6 & 4 & 6 \\
\hline 8D09 & 8 & 8 & 4 & 8 & 4 & 6 & 4 & 2 & 4 \\
\hline 2D12B & 3 & 2 & 2 & 4 & 2 & 3 & 3 & 2 & 4 \\
\hline 1B09 & 4 & 4 & 3 & 3 & 2 & 4 & 4 & 0 & 4 \\
\hline
\end{tabular}

* Peanut aequatoriana type is not listed because only one accession from ICRISAT was tested in this study. 
Table 2 Polymorphic information content (PIC) values of various peanut types from Chinese and ICRISAT mini core collections as revealed by 26 SSR primer pairs

\begin{tabular}{|c|c|c|c|c|c|c|c|c|c|c|}
\hline \multirow{2}{*}{ Primer } & \multirow{2}{*}{$\begin{array}{l}\text { Total } \\
\text { PIC }\end{array}$} & \multicolumn{5}{|c|}{ Mini core collection from China } & \multicolumn{4}{|c|}{ Mini core collection from ICRISAT* } \\
\hline & & fastigiata & vulgaris & hirsuta & hypogaea & Intermediate & fastigiata & vulgaris & peruviana & hypogaea \\
\hline 2A06 & 0.782 & 0.793 & 0.617 & 0.872 & 0.874 & 0.842 & 0.815 & 0.414 & 0.722 & 0.829 \\
\hline 2B10 & 0.466 & 0.580 & 0.282 & 0.490 & 0.000 & 0.375 & 0.464 & 0.561 & 0.500 & 0.392 \\
\hline 2E06 & 0.933 & 0.919 & 0.896 & 0.928 & 0.934 & 0.922 & 0.907 & 0.891 & 0.000 & 0.917 \\
\hline PM436 & 0.678 & 0.486 & 0.633 & 0.472 & 0.511 & 0.227 & 0.56 & 0.421 & 0.000 & 0.693 \\
\hline PM137 & 0.943 & 0.928 & 0.944 & 0.897 & 0.944 & 0.889 & 0.933 & 0.955 & 0.938 & 0.930 \\
\hline $18 \mathrm{C} 05$ & 0.607 & 0.726 & 0.508 & 0.431 & 0.440 & 0.398 & 0.533 & 0.202 & 0.000 & 0.326 \\
\hline $13 \mathrm{~A} 07$ & 0.497 & 0.330 & 0.381 & 0.000 & 0.421 & 0.219 & 0.064 & 0.242 & 0.000 & 0.317 \\
\hline 9E08 & 0.583 & 0.677 & 0.534 & 0.095 & 0.330 & 0.305 & 0.558 & 0.488 & 0.500 & 0.425 \\
\hline 19A05 & 0.234 & 0.497 & 0.161 & 0.000 & 0.101 & 0.000 & 0.498 & 0.305 & 0.500 & 0.131 \\
\hline $15 \mathrm{~F} 12$ & 0.542 & 0.622 & 0.343 & 0.608 & 0.554 & 0.398 & 0.540 & 0.275 & 0.000 & 0.540 \\
\hline $2 \mathrm{~F} 05$ & 0.893 & 0.906 & 0.882 & 0.903 & 0.883 & 0.000 & 0.418 & 0.375 & 0.000 & 0.200 \\
\hline 7H06 & 0.900 & 0.901 & 0.899 & 0.000 & 0.889 & 0.882 & 0.320 & 0.144 & 0.000 & 0.200 \\
\hline $13 \mathrm{~A} 10$ & 0.896 & 0.922 & 0.883 & 0.000 & 0.884 & 0.000 & 0.983 & 0.897 & 0.000 & 0.878 \\
\hline 18B08 & 0.880 & 0.894 & 0.877 & 0.000 & 0.881 & 0.000 & 0.883 & 0.875 & 0.000 & 0.878 \\
\hline 17E01 & 0.907 & 0.933 & 0.897 & 0.903 & 0.895 & 0.000 & 0.945 & 0.951 & 0.984 & 0.970 \\
\hline $16 \mathrm{C} 06$ & 0.930 & 0.942 & 0.918 & 0.921 & 0.901 & 0.907 & 0.989 & 0.959 & 0.906 & 0.934 \\
\hline 15D03 & 0.889 & 0.569 & 0.345 & 0.177 & 0.504 & 0.461 & 0.480 & 0.172 & 0.500 & 0.472 \\
\hline $16 \mathrm{~F} 10$ & 0.639 & 0.642 & 0.514 & 0.608 & 0.657 & 0.664 & 0.624 & 0.651 & 0.500 & 0.564 \\
\hline PM443 & 0.972 & 0.973 & 0.969 & 0.973 & 0.966 & 0.968 & 0.992 & 0.991 & 0.000 & 0.941 \\
\hline $14 \mathrm{H} 06$ & 0.979 & 0.978 & 0.979 & 0.967 & 0.978 & 0.975 & 0.982 & 0.981 & 0.981 & 0.982 \\
\hline 8D09 & 0.989 & 0.988 & 0.988 & 0.987 & 0.987 & 0.986 & 0.952 & 0.941 & 0.938 & 0.895 \\
\hline 2D12B & 0.911 & 0.907 & 0.895 & 0.894 & 0.899 & 0.889 & 0.660 & 0.510 & 0.500 & 0.534 \\
\hline 1B09 & 0.987 & 0.988 & 0.986 & 0.985 & 0.987 & 0.986 & 0.912 & 0.895 & 0.000 & 0.894 \\
\hline Average & 0.784 & 0.761 & 0.679 & 0.548 & 0.719 & 0.529 & 0.708 & 0.663 & 0.345 & 0.665 \\
\hline
\end{tabular}

* Peanut aequatoriana type is not listed because only one accession from ICRISAT was tested in this study.

Table 3 Similarity coefficients of different peanut types from Chinese and ICRISAT mini core collections

\begin{tabular}{|c|c|c|c|c|c|c|c|}
\hline \multirow{2}{*}{ Botanical type } & \multirow{2}{*}{$\begin{array}{l}\text { Number of } \\
\text { genotypes }\end{array}$} & \multicolumn{3}{|c|}{ Similarity coefficient } & \multicolumn{2}{|c|}{ Similarity coefficient no larger than 0.60} & \multirow{2}{*}{$\begin{array}{c}\text { Genetic diversity } \\
\text { index }\end{array}$} \\
\hline & & Maximum & Minimum & Average & Number of genotype pair & Percentage $(\%)$ & \\
\hline \multicolumn{8}{|c|}{ Mini core collection from China } \\
\hline fastigiata & 29 & 0.98 & 0.55 & 0.73 & 18 & 4.43 & 1.11 \\
\hline vulgaris & 119 & 0.99 & 0.57 & 0.82 & 7 & 0.10 & 0.87 \\
\hline hirsuta & 21 & 0.97 & 0.64 & 0.85 & 0 & 0.00 & 0.73 \\
\hline hypogaea & 110 & 0.99 & 0.56 & 0.78 & 30 & 0.50 & 0.89 \\
\hline Intermediate & 19 & 0.99 & 0.71 & 0.85 & 0 & 0.00 & 0.87 \\
\hline Subtotal & 298 & 0.99 & 0.51 & 0.74 & 560 & 1.26 & 0.89 \\
\hline \multicolumn{8}{|c|}{ Mini core collection from ICRISAT } \\
\hline fastigiata & 30 & 0.94 & 0.57 & 0.76 & 4 & 0.88 & 0.97 \\
\hline vulgaris & 64 & 0.99 & 0.61 & 0.82 & - & - & 0.83 \\
\hline peruviana & 2 & - & - & 0.79 & - & - & 0.61 \\
\hline Subtotal & 168 & 0.99 & 0.53 & 0.76 & 367 & 2.59 & 0.82 \\
\hline Total & 466 & 0.99 & 0.49 & 0.74 & 2791 & 2.56 & 0.86 \\
\hline
\end{tabular}

* Peanut aequatoriana type is not listed because only one accession from ICRISAT was tested in this study. 
mini core collection than in the ICRISAT core collection; however, there are more genotypes with large difference in the ICRISAT collection.

The average similarity coefficients of fastigiata and hypogaea types from ICRISAT, which were 0.76 and 0.81 respectively, were larger than those from China $(0.73$ and 0.78); whereas, the similarity coefficients of vulgaris type were almost the same between the 2 mini core collections. In the Chinese collection, fastigiata type presented not only the smallest similarity coefficients but also the second highest percentage of genotype pair with low similarity. In contrast, hirsute and intermediate types showed large similarities among genotypes. Genetic diversity index also revealed that fastigiata type had relatively rich genetic diversity, whereas hirsute and intermediate types were in narrow variation. In the ICRISAT collection, the smallest similarity and largest genetic diversity index were also found in fastigiata type (Table 3).

There were 3 common types of peanut in both mini core collections, i.e., fastigiata, vulgaris, and hypoaea types. After comparing the similarity coefficients of these common types between the Chinese and ICRISAT mini core collections, it was found that similarities among botanical types were smaller in the ICRISAT collection than in the Chinese collection (Table 4). This suggests that the genetic diversity of ICRISAT accessions is mainly contributed by the variations among types rather than variations within types.

\subsection{Similarity between Chinese and ICRISAT mini core collections}

The largest genetic difference was detected between the Chinese accession L2 Gangguo and the ICRISAT accession ICG12625 (aequatoriana type) with the similarity coefficient of 0.49 . ICG12625 also showed small similarities $(<0.51)$ with Chinese accessions Xinbin Silihong, Xinjianshe Huasheng, Longxiwan, Kalimubana, and F2. The genetic difference was the smallest between the Chinese accession Xinhua 6 and the ICRISAT accession ICG1415 with similarity coefficient of 0.94 , followed by genotype pairs of Xinhua 6/ICG1455 (0.93) and 8506-4/ICG4527 (0.93). ICRISAT genotypes ICG12625 and ICG3673 had the largest genetic differences to Chinese genotypes, whose average similarity coefficients were 0.60 and 0.63 , respectively. ICG2925 and ICG2672 were genetically close to the Chinese genotypes with the similarity coefficients as high as 0.76 .

In the 3 common types of both collections, the largest similarity (0.74) was observed between vulgaris types or hypogaea types from different resources. The similarities between the Chinese fastigiata type and the ICRISAT hypogaea type or the Chinese hypogaea type and the ICRISAT fastigiata type were relatively small, with the similarity coefficients of 0.66 . The ICRISAT specific types, aequatoriana and peruviana, had great genetic differences from all of the Chinese types, especially from the Chinese hypogaea type (similarity coefficient of 0.58 ) and the Chinese peruviana type (similarity coefficient of 0.67 ), respectively (Table 5).

\subsection{PCA of Chinese and ICRISAT both mini core collections of peanut}

Based on PCA graph (Figure), the Chinese accessions were mainly involved in groups II, IV, and V with typical genotypes of ssp. hypogaea (hypogaea and hirsuta types), vulgaris type, and fastigiata type, respectively. The ICRISAT accessions were also grouped into 3 sections: I, III, and VI, which were mainly

Table 4 Similarity coefficients among botanical types within a mini core collection of peanut

\begin{tabular}{|c|c|c|c|c|c|c|c|c|c|c|}
\hline \multirow{2}{*}{ Botanical type } & \multicolumn{5}{|c|}{ Chinese mini core collection } & \multicolumn{5}{|c|}{ ICRISAT mini core collection } \\
\hline & fastigiata & vulgaris & hypogaea & hirsuta & Intermediate & fastigiata & vulgaris & hypogaea & aequatoriana & peruviana \\
\hline fastigiata & - & 0.72 & 0.71 & 0.71 & 0.68 & - & 0.73 & 0.67 & 0.61 & 0.71 \\
\hline hypogaea & - & - & - & 0.79 & 0.73 & - & - & - & 0.62 & 0.74 \\
\hline hirsuta & - & - & - & - & 0.76 & - & - & - & - & - \\
\hline aequatoriana & - & - & - & - & - & - & - & - & - & 0.66 \\
\hline
\end{tabular}

Table 5 Similarity coefficients among botanical types between Chinese and ICRISAT mini core collections of peanut

\begin{tabular}{|c|c|c|c|c|c|}
\hline \multirow{2}{*}{ Chinese mini core collection } & \multicolumn{5}{|c|}{ ICRISAT mini core collection } \\
\hline & aequatoriana & peruviana & fastigiata & vulgaris & hypogaea \\
\hline fastigiata & 0.60 & 0.67 & 0.68 & 0.69 & 0.66 \\
\hline vulgaris & 0.62 & 0.71 & 0.70 & 0.74 & 0.71 \\
\hline hirsuta & 0.62 & 0.68 & 0.66 & 0.68 & 0.74 \\
\hline hypogaea & 0.58 & 0.69 & 0.67 & 0.69 & 0.74 \\
\hline Intermediate & 0.63 & 0.73 & 0.68 & 0.73 & 0.74 \\
\hline
\end{tabular}




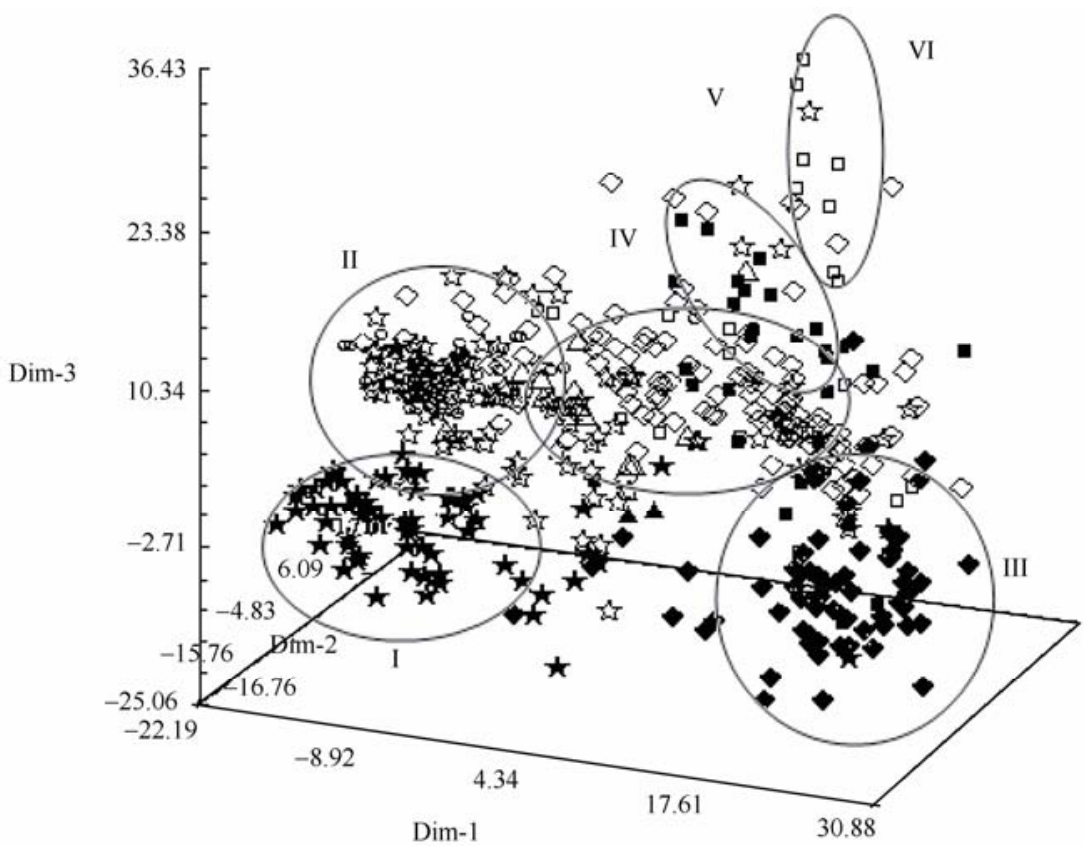

Figure 3D-PCA graph of peanut mini core collections from China and ICRISAT based on SSR analysis Group I is mainly composed of hypogaea type; Group II is mainly composed of hypogaea and hirsuta types; Groups III and IV are mainly implicated with vulgaris type; Groups V and VI are mainly composed of fastigiata type.

composed of hypogaea, vulgaris, and fastigiata types, respectively. Apparently, there were great genetic differences between the Chinese and the ICRISAT mini core collections. The 3 common types from China and ICRISAT were all separated into different groups in the PCA graph. For example, the Chinese and ICRISAT hypogaea types were classified into groups II and I, respectively. The 3 principal components explained $61.22 \%$ of the total genetic variation between the 2 populations, of which $33.48 \%$ for component $1,16.60 \%$ for component 2 , and $11.20 \%$ for component 3 . This result suggests that the ICRISAT germplasm is a valuable supplement to the Chinese peanut resources.

\section{Discussion}

In this study, we revealed that the general genetic diversity in the Chinese mini core collection of peanut was richer than that in ICRISAT mini core collection. However, compared with the Chinese collection, the ICRISAT collection implicated more genotypes with large genetic distances to other genotypes within the population. This finding is consistent with our previous study on the phenotypic traits of the 2 mini core collections ${ }^{[10]}$. The Chinese collection showed a wider variation in botanic characteristics than the ICRISAT collection, such as height of main stem, node number of main stem, leaflet width, number of branch with pods, pod number per plant, pod yield per plant, and shelling percentage; but the variations of total branching number and pot length were larger in the ICRISAT collection than in the Chinese collection ${ }^{[10]}$. The high level of phenotypic diversity of the Chinese collection mainly results from many hirsuta genotypes involved ${ }^{[6,7]}$. Although a few aequatoriana and peruviana genotypes are implicated in the ICRISAT collection, they have similar botanic characteristics to some Chinese fastigiata genotypes, such as leaflet shape, pod reticulation, pod beak, and seed coat color.

Chinese peanut germplasm is classified into subspecies hypogaea and fastigiata. Based on morphological traits and growth habits, the former subspecies is further classified into botanical types hypogaea and hirsuta, and the latter is divided into vulgaris and fastigiata types. The intermediate type is the offspring of cross between subspecies hypogaea and fastigiata. The ICRISAT mini core collection contains genotypes from hypogaea, aequatoriana, peruviana, fastigiata, and vulgaris botanical types and a unique accession of hirsuta type ${ }^{[20]}$, which might be introduced from China. However, this hirsuta genotype was not involved in this study. Types aequatoriana, peruviana, fastigiata, and vulgaris belong to subspecies fastigiata. In both Chinese and ICRISAT mini core collections, fastigiata type had the largest genetic diversity among botanical types as revealed by 26 SSR markers, followed by hypogaea type. This result may provide direct guidance to genetic enhancement and cultivar improvement of peanut.

The PCA result showed remarkable difference on genetic 
background between China and ICRISAT core collections. Except for fastigiata accessions, the genotypes with different sources were separated in different groups in spite of the same botanical type. The unique aequatoriana genotype ICG12625 had great genetic differences to L2 Gangguo, Xinbin Silihong, Xinjianshe Huasheng, and Longxiwan. These unique genotypes are good materials to construct mapping populations and genetic linkage maps.

\section{Conclusions}

The level of genetic diversity was higher in the Chinese mini core collection than in the ICRISAT mini core collection. Relatively large variation was detected in fastigiata type, followed by hypogaea type. The least genetic similarity was found between the Chinese genotype L2 Ganguo and the ICRISAT accession ICG12625.

\section{Acknowledgments}

This study was supported by the National Key Technologies R\&D Program of China (2006BAD13B05-2), the National Natural Science Foundation of China (30571132), the National Basic Platform for Science and Technology Research of China (2005DKA21002-13), and the project of Protection of Crop Germplasm Resources (NB07-2130135-35).

\section{References}

[1] Wang Y B, Zhang Y B, Zhang P, Men A J. Perspectives and export promoting strategies in Chinese peanut industry after entering WTO. J Peanut Sci, 2003, 32(suppl): 24-29 (in Chinese)

[2] Liao B S. Competitiveness analysis of oil industry in China. $J$ Peanut Sci, 2003, 32(suppl): 11-15 (in Chinese)

[3] Liang X Q, Pan R Z, Bin J H. Progress on mechanism of resistance to Aspergillus infection in peanut. Chin Oil Crops Sci, 2000, 22(3): 77-80 (in Chinese with English abstract)

[4] Jiang H F, Wang S Y, Ren X P. Reaction of groundnut germplasm to Aspergillus flavus invasion. Chin Oil Crops Sci, 2002, 24(1): 23-25 (in Chinese with English abstract)

[5] Yu S L. Pedigree of Peanut Variety in China. Shanghai: Shanghai Scientific and Technical Publishers, 2008 (in Chinese)

[6] Sun D R. Peanut Breeding. Beijing: China Agriculture Press, 1998 (in Chinese)

[7] Wan S B. Peanut Cultivation in China. Shanghai: Shanghai Scientific and Technical Publishers, 2008 (in Chinese)

[8] Jiang H F, Ren X P. Genetic diversity of peanut resource on morphological characters and seed chemical components in China. Chin Oil Crops Sci, 2006, 28: 421-426 (in Chinese with English abstract)
[9] Jiang H F, Ren X P, Huang J Q, Liao B S, Lei Y. Establishment of peanut mini core collection in China and exploration of new resource with high oleat. Chin Oil Crops Sci, 2008, 30: 294-299 (in Chinese with English abstract)

[10] Jiang H F, Ren X P, Liao B S, Huang J Q, Lei Y, Chen B Y, Guo B Z, Holbrook C C, Upadhyaya H D. Peanut core collection established in china and compared with ICRISAT mini core collection. Acta Agron Sin, 2008, 34: 25-30 (in Chinese with English abstract)

[11] Mace E S, Phong D T. SSR analysis of cultivated groundnut (Arachis hypogaea L.) germplasm resistant to rust and late leaf spot diseases. Euphytica, 2006, 152: 317-330

[12] Upadhyaya H D. Phenotypic diversity in groundnut (Arachis hypogaea L.) core collection assessed by morphological and agronomical evaluations. Genet Resour Crop Evol, 2003, 50: $539-550$

[13] Upadhyaya H D, Bramel P J, Ortiz R, Singh S. Geographical patterns of diversity for morphological and agronomic traits in the groundnut germplasm collection. Euphytica, 2002, 128: 191-204

[14] Jiang H F, Liao B S, Ren X P, Lei Y, Mace E, Fu T D, Crouch J H. Comparative assessment of genetic diversity of peanut (Arachis hypogaea L.) genotypes with various levels of resistance to bacterial wilt through SSR and AFLP analyses. $J$ Genet Genomics, 2007, 34: 544-554

[15] Jiang H F, Chen B Y, Ren X P, Liao B S, Lei Y, Fu T D, Ma C Z, Mace E, Crouch J H. Identification of SSR markers linked to bacterial wilt resistance of peanut with RILs. Chin Oil Crops Sci, 2007, 29: 26-30 (in Chinese with English abstract)

[16] Ferguson M E, Bramel P J, Chandra S. Gene diversity among botanical varieties in peanut (Arachis hypogaea L.). Crop Sci, 2004, 44: 1847-1854

[17] Liu J, Guan J P, Xu D X, Zhang X Y, Gu J, Zong X X. Analysis of genetic diversity and population structure in lentil (Lens culinaris Medik.) germplasm by SSR markers. Acta Agron Sin, 2008, 34: 1901-1909 (in Chinese with English abstract)

[18] Zong X X, Guan J P, Wang S M, Liu C Q. Genetic diversity among Chinese pea (Pisum sativum L.) landraces revealed by SSR markers. Acta Agron Sin, 2008, 34: 1330-1338 (in Chinese with English abstract)

[19] Yu Y, Wang Z W, Feng C H, Zhang Y X, Lin Z X, Zhang X L. Genetic evaluation of EST-SSRs derived from Gossypium herbaceum. Acta Agron Sin, 2008, 34: 2085-2091 (in Chinese with English abstract)

[20] Upadhyaya H D, Bramel P J, Ortiz R, Singh S. Developing a mini core of peanut for utilization of genetic resources. Crop Sci, 2002, 42: 2150-2156

[21] Holbrook C C, Anderson W F, Pittman R N. Selection of a core collection from the US germplasm collection of peanut. Crop Sci, 1993, 33: 859-861

[22] Holbrook C C, Dong W B. Developing and evaluation of a mini core collection for the US peanut germplasm collection. Crop Sci, 2005, 45: 1540-1544 